\title{
Doctors' experience of a bespoke physician consultation service: cross-sectional investigation
}

\author{
Maja Meerten, ${ }^{1}$ Julia Bland, ${ }^{2}$ Samantha R. Gross, ${ }^{2}$ Antony I. Garelick ${ }^{1}$
}

The Psychiatrist (2011), 35, 206-212, doi: 10.1192/pb.bp.110.031047

${ }^{1}$ Tavistock \& Portman NHS Trust London; ${ }^{2}$ Institute of Psychiatry, King's College London

Correspondence to Antony I. Garelick (AGarelick@tavi-port.nhs.uk)

First received 7 May 2010, final revision 6 Sep 2010, accepted 19 Nov 2010

\begin{abstract}
Aims and method Our aim was to follow-up on a cohort of self-referred doctors who attended MedNet. We used a two-point cross-sectional design. Measures included three standardised self-report questionnaires administered before and after consultation. Doctors were also asked to complete a service user questionnaire, and data regarding engagement and onward referrals were gathered through case-note review.

Results A statistically significant improvement in scores on all three questionnaires was found after intervention; however, scores on one subscale, the risk domain of the Clinical Outcomes in Routine Evaluation - Outcome Measure, did not change significantly. Of the doctors at no risk of suicide at intake, nearly two-thirds ( $n=41 / 70,59 \%)$ were sufficiently helped by the consultations provided to not need further treatment. Of the doctors at some risk of suicide at intake, two-thirds $(n=34 / 51,67 \%)$ did need an onward referral. Only one doctor required hospital admission, an outcome that suggests the approach used is containing and clinically responsive.
\end{abstract}

Clinical implications This paper highlights the efficacy, need and importance of specialist services for doctors in difficulty. We found that the bespoke consultation model provided at MedNet is valued highly by the doctors as service users.

Declaration of interest A.I.G. is the Associate Dean, MedNet, London Deanery.
It has been reported that compared with the general population, doctors are more likely to experience high levels of stress and mental ill health. ${ }^{1}$ When doctors are unwell this has adverse effects on the level of patient care, on the doctor and on the organisation in terms of cost. ${ }^{2}$ It is important to acknowledge that it is often the best doctors who succumb and that one cannot afford to lose them from the profession. ${ }^{3}$ The worldwide shortage of doctors makes their health especially important for recruitment and retention to the medical profession. ${ }^{4}$

Research has shown that doctors often undertake selftreatment when unwell and may 'work through an illness' ('presenteeism'). ${ }^{5,6}$ Doctors will generally try to 'sort themselves out' first, and are less likely to find their way to appropriate services. Much of this can be as a result of denial and minimisation of their own symptoms, and also because of additional difficulties they may experience in taking on the role of patient. ${ }^{5}$

The recently published report by the Department of Health, Mental Health and Ill Health in Doctors, recognises the reluctance doctors may have in seeking help through the combined forces of stigma, shame and secrecy, together with fear of damage to their professional status. The report also highlights the importance of promoting mental health and well-being in doctors and developing accessible, appropriate services in order to meet their needs. ${ }^{7}$

\section{MedNet}

The MedNet service was created in 1997 and is funded by the London Deanery in order to provide doctors of all grades with strictly confidential consultations informed by psychodynamic principles. Although MedNet is a selfreferral service, many doctors report being prompted to make contact by others (colleagues, occupational health, general practitioners etc.), which indicates that problems have become severe enough to be visible. MedNet is staffed by consultant psychiatrists in psychotherapy, who have seen over 750 doctors face to face in the past 10 years. The model is of an extended consultation process of up to six sessions. The consultations embrace a broad range of issues from medical practice, work-life balance and personal difficulties with which practitioners are struggling, and at the same time offer the opportunity to explore possible internal conflicts. When needed, access to further expert help is facilitated, which may consist of more specialised psychotherapeutic interventions, embracing all the mainstream modalities of psychotherapy or specialised psychiatric advice. In most cases the standard consultation 
of up to six sessions is sufficient to either complete the intervention or agree that ongoing psychological help elsewhere is appropriate, but for a limited number of doctors, longer-term consultation or psychological treatment is provided.

\section{Previous paper}

A previous paper from this group described the characteristics of a cohort of 121 doctors who presented to the MedNet service between 2002 and $2004 .^{8}$ The most prevalent presenting problems included depression, anxiety, interpersonal difficulties and work-related issues. Only $9 \%$ of the cohort were identified as severely distressed psychiatrically using Brief Symptom Inventory (BSI) measures. ${ }^{9}$ In approximately half the sample, problems first presented in the year preceding presentation. ${ }^{8}$ A total of $42 \%$ were considered to be at some risk of suicide, more than a quarter of whom were considered to have a moderate to severe risk. ${ }^{8}$

The majority of follow-up reports have examined doctors presenting to services with substance use disorders, ${ }^{10}$ whereas the MedNet service captures a different population. At MedNet, doctors predominantly present with burnout, fatigue, depression, anxiety and suicidal ideation. ${ }^{8}$ Specialist services for doctors with these kinds of difficulties have also been established and researched in the Villa Sana Resource Centre in Norway ${ }^{11}$ and the Galatea Clinic in Spain. $^{12}$

In this paper, we present follow-up data from the cohort of 121 self-referred doctors in order to assess the impact of a series of face-to-face consultation sessions at the service in terms of outcomes, to evaluate the doctors' personal experiences of having attended MedNet, and to report referrals made onward from the service as a result of the consultation process.

\section{Method}

\section{The sample}

All doctors who contacted the service between February 2002 and February 2004 were eligible for inclusion $(n=123)$. Of these, $98.4 \%(n=121)$ consented to take part.

\section{Measures}

Data were gathered from three standardised self-report questionnaires: the Clinical Outcomes in Routine Evaluation - Outcome Measure (CORE-OM) ${ }^{13}$ the BSI $;^{9}$ the Maslach Burnout Inventory (MBI); ${ }^{14}$ and a 20 -item bespoke questionnaire designed by the service to assess users' views and experiences of having attended. At intake a CORE therapist assessment questionnaire was also completed, but this was not repeated at follow-up. The standardised selfreport questionnaires were completed at two points in time (before being seen at the service and at the end of the consultation process) with the service user questionnaire administered only at follow-up. The CORE-OM is a 34-item, 5 -point Likert scale questionnaire that provides scores for the subscales of subjective well-being, commonly experienced problems or symptoms, life/social functioning
}

and risk to self and others. Standardised scores to determine clinical cut-off points on this measure were used as provided by the distributor. ${ }^{13}$ The BSI is a 53-item, 5 -point Likert scale questionnaire that targets symptoms in 9 areas. Caseness implies the criterion for requiring a formal mental health assessment and was computed using adult gender-matched non-patient norms. Adult psychiatric outpatient norms for the BSI and the Global Severity Index (GSI) as a component of the BSI were used to measure clients' degree of symptomatic distress. ${ }^{9}$ The MBI is a 22 item, 7-point Likert scale questionnaire that evaluates workrelated distress through three dimensions: emotional exhaustion, depersonalisation and personal accomplishment, each of which was scored independently. ${ }^{14}$ The user questionnaire is a service-specific, 20-item, 4-point Likert scale questionnaire combined with a section of open boxes to reflect service users' comments and suggestions. Followup questionnaires were administered by post.

Data regarding engagement with and referral on from the service were gathered by two of the authors through examining the notes. This included data regarding frequency of being seen at the service, whether the doctors were on medication prior to presentation to the service, the number who completed a treatment episode (in the service) and did not need further treatment, how many were referred on for additional treatment and for which type of further treatment. The treatment that doctors engaged with and were advised on were compared in terms of the subsample of suicidal and non-suicidal doctors based on CORE therapist assessment at intake as described in the original paper. 8

Data collection and analysis at baseline are described in detail in the original paper The scores of those who completed the questionnaires at follow-up were analysed using paired sample $t$-tests. Quantitative data were analysed using SPSS v. 15 for Windows. Data regarding engagement, onward referral and user satisfaction were analysed thematically by two of the authors.

\section{Results \\ CORE-OM}

In total 121 consented to take part in the study. Of these, 95\% $(n=115)$ completed the CORE-OM at intake, $74 \%$ $(n=89)$ completed it post-treatment and 73\% $(n=86)$ fully completed both pre- and post-treatment questionnaires. There were no inter-group differences in terms of the doctors who did and did not complete the follow-up questionnaire.

Significant reductions for all domains on the COREOM were identified between pre- and post-treatment, apart from the domain of risk (Table 1). There was a reduction in the proportion of doctors who scored above clinical cut-off on the CORE-OM at follow-up ( $>1.19$ male, $>1.29$ female), from $63 \%$ ( $n=72 / 115)$ of respondents to $34 \%(n=30 / 89)$. Of the 86 individuals who completed the questionnaire both pre- and post-intervention, 52 had scored above clinical cut-off at intake, and more than half $(n=28 / 52)$ no longer had a high score at follow-up. However, six individuals with 


\begin{tabular}{|c|c|c|c|c|}
\hline \multirow[b]{2}{*}{ Subscale } & \multirow{2}{*}{$\begin{array}{l}\text { Pre-intervention, mean (s.d.) } \\
\qquad(n=115)\end{array}$} & \multirow{2}{*}{$\begin{array}{l}\text { Post-intervention, mean (s.d.) } \\
\qquad(n=89)\end{array}$} & \multicolumn{2}{|c|}{ Paired samples $(n=86)$} \\
\hline & & & $t$-test & $P$ \\
\hline Functioning & $1.41(0.76)$ & $1.05(0.85)$ & 3.98 & $<0.001$ \\
\hline Problems & $1.84(0.86)$ & $1.22(0.93)$ & 6.36 & $<0.001$ \\
\hline Well-being & $2.02(0.97)$ & $1.28(1.09)$ & 6.44 & $<0.001$ \\
\hline Risk & $0.32(0.48)$ & $0.27(0.50)$ & 0.418 & 0.677 \\
\hline Total score & $1.44(0.69)$ & $1.00(0.80)$ & 5.58 & $<0.001$ \\
\hline
\end{tabular}

a normal score at intake were above clinical cut-off at follow-up.

For the subsample of doctors with suicidal risk prior to treatment ( $n=51, n=35$ completed at both time points), there remained a significant difference in scores on all subscales and the CORE-OM overall score for these domains, apart from risk. Whereas 34 of the subsample were above cut-off pre-intervention, only 16 were above cutoff post-intervention ( 3 new clients, 14 were no longer above cut-off).

\section{BSI}

Of the initial 121 doctors, $94.2 \%(n=14)$ completed the BSI questionnaire prior to treatment, $75.2 \%(n=91)$ completed the questionnaire at follow-up and $72.7 \%(n=88)$ completed both pre- and post-treatment questionnaires. There were no inter-group differences in terms of the doctors who did and did not complete the follow-up questionnaire. There was a significant difference between all BSI subscales taken pre-and post-treatment, excluding phobic anxiety (Table 2).

Whereas $85 \%(n=97 / 114)$ of responding doctors scored above clinical cut-off for non-patient norms on the BSI at intake (indicating the individual may require a formal mental health assessment), this number reduced to $51.6 \%$
( $n=48 / 91$ ) at follow-up. Of those who completed the questionnaire both pre- and post-treatment, 32 (of 42) individuals who had scored above cut-off, no longer fell within this group. Only eight doctors scored above clinical cut-off for psychiatric out-patients at follow-up, a reduction of three individuals; all had scored above cut-off at intake.

\section{MBI}

Of the initial 121 doctors, $94.2 \%(n=114)$ completed the MBI questionnaire prior to treatment, $67 \% \quad(n=81)$ doctors completed the questionnaire at follow-up and $65.3 \%$ $(n=79)$ completed both pre- and post-treatment questionnaires. There were no inter-group differences in terms of the doctors who did and did not complete the follow-up questionnaire. There was a significant change in scores for the emotional exhaustion subscale of the MBI at follow-up ( $t=5.55, P<0.001$ ), but not for the remaining subscales of depersonalisation and personal achievement (Table 3 ).

\section{Engagement with and referral on from the service}

Of the 121 doctors included in the study, 33.9\% $(n=41)$ were on medication, the majority on antidepressants (85\%, $n=35$ ). Most doctors were seen for up to six sessions

Table 2 Brief Symptom Inventory outcomes

\begin{tabular}{|c|c|c|c|c|}
\hline \multirow[b]{2}{*}{ Subscale } & \multirow{2}{*}{$\begin{array}{l}\text { Pre-intervention, mean (s.d.) } \\
\qquad(n=114)\end{array}$} & \multirow{2}{*}{$\begin{array}{l}\text { Post-intervention, mean (s.d.) } \\
\qquad(n=91)\end{array}$} & \multicolumn{2}{|c|}{ Paired samples $(n=88)$} \\
\hline & & & $t$-test & $P$ \\
\hline Somatisation & $0.44(0.62)$ & $0.30(0.54)$ & 2.50 & 0.014 \\
\hline Obsessive-compulsive disorder & $1.42(0.95)$ & $0.94(0.95)$ & 4.59 & $<0.001$ \\
\hline Interpersonal sensitivity & $1.36(1.01)$ & $1.09(1.10)$ & 3.00 & 0.004 \\
\hline Depression & $1.48(0.98)$ & $0.94(0.99)$ & 5.29 & $<0.001$ \\
\hline Anxiety & $1.13(0.94)$ & $0.76(0.87)$ & 3.61 & 0.001 \\
\hline Hostility & $0.74(0.75)$ & $0.53(0.67)$ & 2.64 & 0.010 \\
\hline Phobic anxiety & $0.45(0.74)$ & $0.32(0.59)$ & 1.85 & $\begin{array}{l}0.068 \\
\text { (n.s.) }\end{array}$ \\
\hline Paranoid ideation & $0.92(0.86)$ & $0.70(0.77)$ & 2.57 & 0.012 \\
\hline Psychoticism & $0.81(0.67)$ & $0.54(0.65)$ & 4.19 & $<0.001$ \\
\hline Global Severity Index & $0.98(0.65)$ & $0.67(0.66)$ & 4.97 & $<0.001$ \\
\hline Positive Symptom Distress Index & $26.33(10.53)$ & $1.53(0.53)$ & 4.86 & $<0.001$ \\
\hline Positive Symptom, total & $1.83(0.57)$ & $19.60(14.20)$ & 5.55 & $<0.001$ \\
\hline
\end{tabular}

n.s., not significant. 
Table 3 Maslach Burnout Inventory outcomes

\begin{tabular}{|c|c|c|c|c|}
\hline \multirow[b]{2}{*}{ Subscale } & \multirow{2}{*}{$\begin{array}{l}\text { Pre-intervention, mean (s.d.) } \\
\qquad(n=114)\end{array}$} & \multirow{2}{*}{$\begin{array}{l}\text { Post-intervention, mean (s.d.) } \\
\qquad(n=81)\end{array}$} & \multicolumn{2}{|c|}{ Paired samples $(n=79)$} \\
\hline & & & $t$-test & $P$ \\
\hline Emotional exhaustion & $31.97(11.87)$ & $26.14(12.26)$ & 5.55 & $<0.001$ \\
\hline Depersonalisation & $9.08(6.73)$ & $8.55(6.64)$ & 0.92 & 0.358 \\
\hline Personal achievement & $34.59(7.59)$ & $35.07(8.58)$ & -0.69 & 0.493 \\
\hline
\end{tabular}

(78.5\%, $n=95)$; however, $16.5 \%(n=20)$ were seen for $6-20$ sessions and $5 \%(n=6)$ for more than 20 sessions.

Doctors who presented with suicidal risk according to the CORE-OM at intake were compared with those who presented as non-suicidal with regard to their engagement with the service and referral onward. Within this analysis we examined how many sessions an individual attended and to what type of treatment they were referred on to, should they have received an onward referral (Fig. 1).

Of the $70(58 \%)$ doctors who were not deemed suicidal at intake, $58.6 \%(n=41)$ were sufficiently helped by the process of being seen for consultation at MedNet alone, $1.4 \%(n=1)$ was seen for a prolonged episode of treatment at MedNet and $34.3 \%(n=24)$ were referred on for further treatment.

Of the 51 (42\%) doctors who were deemed at some risk of suicide at the initial consultation, $18 \%(n=9)$ were sufficiently helped by being seen at MedNet, $10 \%(n=5)$ were seen for a prolonged episode of treatment at MedNet and $66.7 \%(n=34)$ were referred on for further treatment.

Despite the high level of suicidal ideation in the cohort, only one doctor was admitted to hospital. This was an emergency informal admission to a National Health Service (NHS) hospital because of the degree of suicidal risk at presentation. There were eight $(6.6 \%)$ further referrals to NHS services: psychiatric out-patient clinics (six), an eating disorder service (one), and a therapeutic community (one).

\section{Service user satisfaction}

Of the initial 121 doctors, $83.5 \%(n=101)$ returned the questionnaire, $78.5 \%(n=95)$ completed the full questionnaire and $5 \%(n=6)$ completed only part of the questionnaire. In terms of the clinical service provided, doctors were asked to rate their satisfaction-dissatisfaction with the service on a four-point Likert scale on a number of servicespecific questions as shown in Table 4.

Furthermore, they were asked for their views in terms of the practical side of the service provision such as transport links and accessibility of the service, comfort of the waiting room, helpfulness of reception staff, etc., and were asked to comment on these matters in open boxes and offer suggestions in terms of ways in which the service could be improved. The main themes that emerged from the open boxes are described in Box 1 .

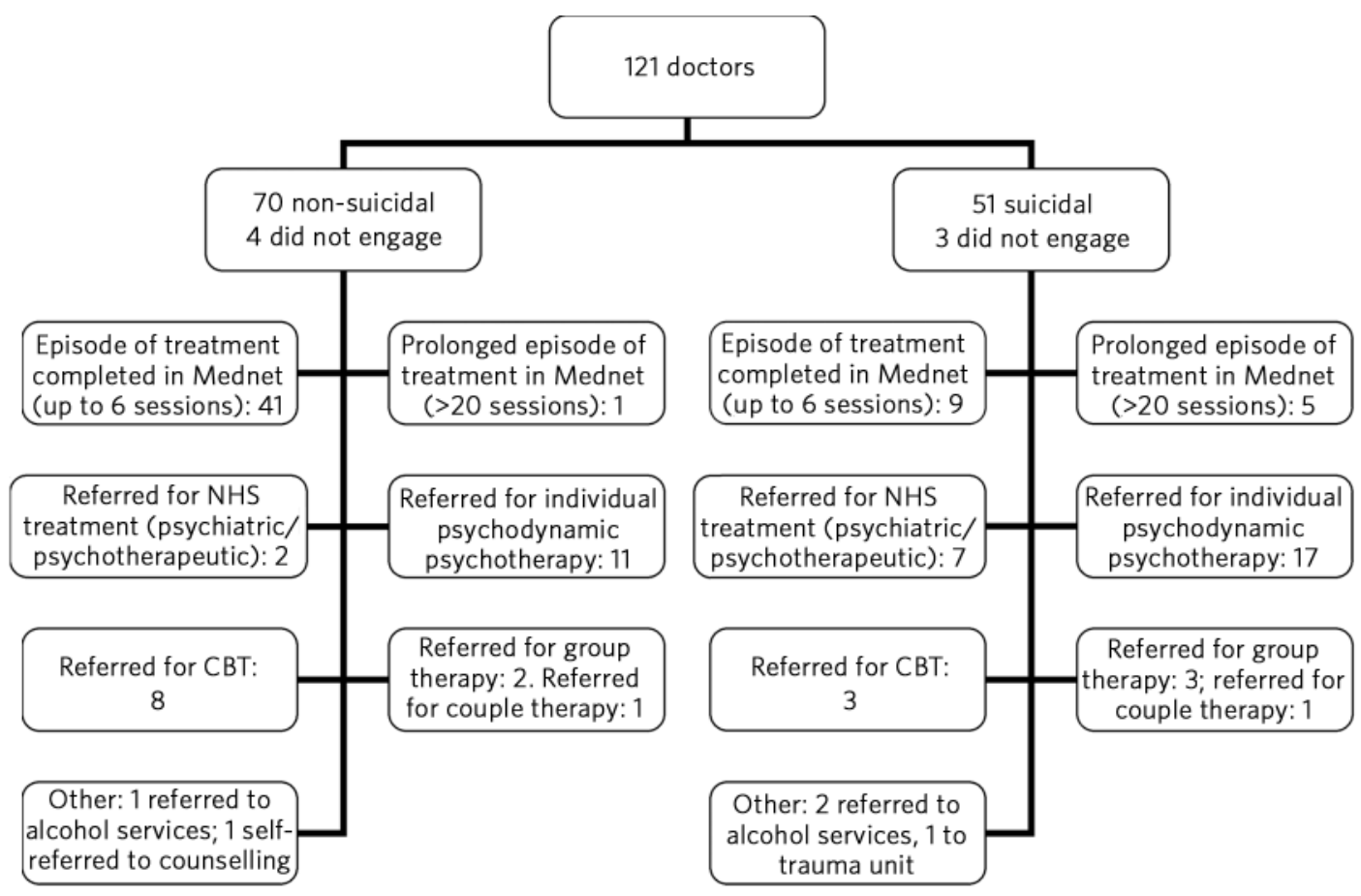

Fig 1 The cohort's treatment and referral pathways.

CBT, cognitive-behavioural therapy; NHS, National Health Service. 
Table 4 Service user questionnaire outcomes

\begin{tabular}{|c|c|c|c|c|}
\hline How satisfied/dissatisfied were you that & $\begin{array}{c}\text { Very satisfied } \\
n(\%)\end{array}$ & $\begin{array}{l}\text { Satisfied } \\
n(\%)\end{array}$ & $\begin{array}{c}\text { Dissatisfied } \\
n(\%)\end{array}$ & $\begin{array}{c}\text { Very dissatisfied } \\
n(\%)\end{array}$ \\
\hline The sessions were allowing you to talk about your problems & $70(70)$ & $27(27)$ & $2(2)$ & $1(1)$ \\
\hline The sessions were helping you understand your problems & $51(50.5)$ & $41(41.4)$ & $6(6.1)$ & $1(1.1)$ \\
\hline The sessions were enabling you to cope with your problems & $38(38.8)$ & $49(50)$ & $9(9.2)$ & $2(2)$ \\
\hline The sessions were addressing important issues to you & $48(48)$ & $44(44)$ & $6(6)$ & $2(2)$ \\
\hline The clinician treated you with respect & $83(82.2)$ & $17(16.8)$ & - & $1(1)$ \\
\hline Information about you and your problems would be confidential & $58(61)$ & $34(37.8)$ & $3(3.1)$ & - \\
\hline
\end{tabular}

\section{Box 1 Service user comments and suggestions}

\section{Comments regarding treatment at the clinic ( $n$ )}

- The service received was excellent (30)

- Would have liked more sessions (8)

- Feeling worse afterwards by stirring up effect of sessions (3)

- Difficult to get here/take time off (3)

Comments regarding service improvement $(n)$

- The service needs better publicity (5)

- Evening appointments should be made available (5)

- Longer treatment and more frequent appointments should be made available (5)

- Better accessibility/improved clinic and parking information would be useful (5)

- More information about the six-session assessment process including confidentiality (4)

\section{Discussion}

\section{MedNet outcomes}

In this naturalistic study, the instruments used demonstrated a significant difference between before and after treatment scores on all questionnaires.

When we examined the results of the suicidal sample, on the measures used there was no significant improvement in terms of risk. However, clinically, in this group, during the time of the study, no suicide attempts or overdoses occurred. The most common clinical dynamic in this group is of doctors who were perfectionists and had set high standards for themselves, with a self-punitive stance if these were not achieved. In psychodynamic terms a possible understanding of the high rate of suicidal ideation is that these doctors have a rejecting or attacking response to their own vulnerabilities, which they see as indicative of failure and weakness. Frequently an associated belief is that evidence of vulnerability or of a need for help is an impediment on their ability to maintain high standards, in particular in their professional lives. In general, these are high-functioning individuals who tend to keep working very well, at the expense of their personal relationships and despite significant mental distress. Often these doctors would not present as globally depressed and have waited a long time to seek help. Our cohort consisted, on the whole, of doctors who had had either none or minimal contact with services prior to being seen at Mednet. We would suggest that the type of broad assessment provided at MedNet, within an analytic framework, helped these doctors to be open about difficulties they had previously not declared or discussed. Being able to express, discuss and understand thoughts felt to be forbidden, including suicidal thoughts, contributed to a reduction of mental distress. In addition, becoming able to have a conceptual understanding of their difficulties gave them a view that they could be helped. It is important to recognise that symptoms - which showed marked change in terms of formal outcomes over the period of the study - may need longer-term psychotherapeutic work if improvement is to be maintained and resilience to further difficulty promoted (as discussed under care pathways).

With regard to the MBI, there was a significant change only for the emotional exhaustion subscale. This is the domain that we would expect to show change first, both in the development of burnout and its improvement, rather than the more entrenched domains of personal achievement and depersonalisation. A paper by Rø et $a l^{11}$ reported similar findings when researching a group of doctors participating in the Norwegian Villa Sana counselling programme. In this paper, emotional exhaustion was seen as the primary dimension in the subsequent development of burnout. The apparent discrepancy in the Sana doctors between reporting high levels of emotional exhaustion, and at the same time not demonstrating reduced empathy or work capacity was thought to suggest that either they take action in time to prevent burnout or stay at work as 'hazardous heroes', even with considerable distress, in order to fulfil their obligations and to maintain their self-esteem. ${ }^{11}$

\section{Engagement with and referral on from the service}

In terms of the pathways of treatment and referral, about three-quarters of doctors were seen for up to 6 sessions, a further $15 \%$ were seen for up to 20 sessions and $5 \%$ were seen for more than 20 sessions.

Two-thirds of doctors who were not suicidal were helped by the treatment provided at MedNet and found it sufficient, whereas the remainder were referred on for additional treatment. For the suicidal subsample, the service was sufficient for about a quarter. For two-thirds the service was helpful in terms of managing the presenting crisis, feeling listened to, and as a stepping-stone in terms of longer treatment that they were advised about and in many 
cases facilitated in accessing. These results show that the doctors who, according to the CORE-OM and BSI were more depressed and had more significant problems in terms of suicidal ideation, did require more long-term interventions and were therefore more likely to receive longer treatment at Mednet and/or be referred on for further treatment.

Overall, about half of the doctors were helped sufficiently by the treatment that the service provided and of the other half who did need further treatment only one was admitted to hospital. This suggests that the therapeutic relationship had a holding effect and that the service and the approach used managed this special client population effectively.

\section{The MedNet consultation model}

The specific patient population of doctors and the type of problems they present calls for a specialised approach when treating their psychological difficulties. In a similar spirit, Harrison argues in a recent paper that the changing role of doctors and new concepts of medical professionalism call for a bespoke model of assessment of doctors' health and fitness to practise that can take all the relevant aspects into account. $^{15}$

In terms of understanding the complex interplay between work, stress and mental health for doctors, there are three main components to be considered. First, there are the specific components of clinical anxiety and the impact of constantly managing ill patients, as well as coping with the complex organisational dynamics of the working environment. These can generate stress and anxiety that can escalate significantly and may also lead to depression. Second, this brings up questions of the work-life balance that impinge on the personal relationships of doctors, with partners, children and wider family. Third, there is the component of underlying personality, particularly perfectionist traits. A degree of obsessiveness is common among doctors and can help to make them both conscientious and committed but if excessive can become problematic. ${ }^{4}$ All three components need to be considered when dealing with doctors' difficulties and problems with their health.

For the large majority of doctors, working as a doctor provides satisfaction and is an integral part of their personal identity and self-esteem. These aspects of work protect and enhance mental health and the capacity to bear the painful realities that practitioners encounter in their work. The opportunity to seek understanding of one's role as a doctor and factors that lead to professionals losing touch with this aspect of their work may also be part of the consultation process. $^{16}$

The MedNet service therefore promotes a narrativebased, holistic approach to doctors' problems, embracing the different issues they may face in terms of clinical practice, organisational stressors, work-life balance, personal characteristics, intrapersonal conflicts and interpersonal difficulties. In this ethos, the service provides consultations in a broad-ranging way in contrast to a more focused diagnostic-based medical model approach. The process of a series of consultations enables engagement and the gradual evolution of an in-depth understanding of a doctor's presenting difficulties.
In order to provide this type of consultation, we believe that expertise in medical and psychiatric fields as well as psychodynamic knowledge of intrapsychic processes and organisational processes is crucial. Doctors often appreciate seeing someone whom they can expect to understand their working life and pressures from 'the inside'.

\section{Service user satisfaction}

The self-report questionnaires show that service users were happy overall with the approach used, and highlighted the importance of the existence of such a service. The questionnaires confirm that the doctors were hoping to have a greater understanding of what generated their difficulties so that they could feel more in control, and more confident that they could manage difficulties when confronted with them again. Wanting to feel in charge of one's own destiny is a particularly strong characteristic in doctors, which is also reflected in the way they are trained. The lengthy pursuit of medical knowledge reinforces the idea that knowledge confers power, prestige, self-worth and a means to autonomy. ${ }^{17}$ Traditionally, doctors have avoided mental health services because of attitudes formed in medical school, a perceived lesser importance of mental health as well as a tendency to treat oneself. ${ }^{18}$ Accordingly, about a third of the doctors attending MedNet had made their own diagnosis, initiated medical treatment in the form of antidepressants and only presented at the service when this approach was ineffective.

The questionnaires also show that information about the confidentiality of the service is an important area for doctors. This is further supported by a recent survey published by the Royal College of Physicians that shows that only one in five doctors would seek professional advice for mental health issues they experience and that the apparent lack of confidence in the current system protecting doctors' confidentiality may act as a barrier to seeking help. $^{18}$

\section{Limitations}

Limitations to our study are that there is no control group in terms of the approach used and service provided at MedNet; response bias; and that data were based on selfreport measures only. However, we hope that despite this we have described usefully the experience of 'doctors in difficulty' through a specialist service and outlined the underlying principles informing the model provided.

\section{Implications}

The observational data show that the mental health of this cohort of doctors has improved. In terms of treatment and referral pathways we found that those who were deemed at some risk of suicide were more likely to have been referred on for further treatment than those who were not at risk, and that only one doctor required admission to hospital. For the more severely unwell doctors, the service was helpful in terms of managing the presenting crisis and feeling heard, and the process was a stepping-stone in terms of longer treatment. Furthermore, we found that the bespoke consultation model provided at MedNet is valued highly 
by the doctors as service users. This paper highlights the efficacy, need and importance of specialist services for doctors in difficulty.

\section{Acknowledgements}

We would like to thank the clients of Mednet for their participation in the study, Mrs Chrystalla Loizou for her highly valued, generous support and help during the preparation of this paper and Dr Elizabeth Gibb for her thorough and substantial manuscript revision. We also thank Professor Elizabeth Paice of the London Deanery for her encouragement and many valuable comments during manuscript preparation.

\section{About the authors}

Maja Meerten is Specialist Trainee in Psychotherapy, Forest House NE London Foundation Trust and Tavistock Clinic, London. Julia Bland is Consultant Psychiatrist and Psychotherapist, MedNet and South London \& Maudsley NHS Foundation Trust. Samantha R. Gross is Senior Addiction Research Worker at the National Addiction Centre, Institute of Psychiatry, King's College London. Antony I. Garelick is the Associate Dean, MedNet Service for Doctors, Tavistock \& Portman NHS Trust, and Forest House NE London Foundation Trust, London.

\section{References}

1 Firth-Cozens J. Doctors, their wellbeing, and their stress. BMJ 2003; 326: 670-1.

2 Firth-Cozens J. A perspective on stress and depression. In Understanding Doctors' Performance (eds J Cox, J King, A Hutchinson, P McAvoy). Radcliffe Publishing, 2006.

3 Bland P. Supporting doctors with mental health problems. Practitioner 2008; 252: 1705-6.

4 Wallace JE, Lemaire JB, Ghali WA. Physician wellness: a missing quality indicator. Lancet 2009; 374: 1714-21.

5 Tyssen R. Health problems and the use of health services among physicians: a review article with particular emphasis on Norwegian studies. Industrial Health 2007; 45: 599-610.
6 Department of Health. NHS Health and Well-Being Review. Boorman Final Report. Department of Health, 2009 (http://www.dh.gov.uk/en/ Publicationsandstatistics/Publications/PublicationsPolicyAnd Guidance/DH_108799).

7 Department of Health. Mental Health and III Health in Doctors. Department of Health, 2008 (http://www.dh.gov.uk/en/Publicationsand statistics/Publications/PublicationsPolicyAndGuidance/DH_083066).

8 Garelick Al, Gross SR, Richardson I, von der Tann M, Bland J, Hale R. Which doctors and with what problems contact a specialist service for doctors? A cross sectional investigation. BMC Med 2007; 5: 26.

9 Derogatis LR. Brief Symptoms Inventory - Administration, Scoring and Procedures Manual (3rd edn). National Computer Services, 1993.

10 Gross SR, Wolff K, Strang J, Marshall EJ. Follow-up of provision of inpatient treatment for UK healthcare professionals with alcohol dependence: snapshot of a pilot specialist national health service. Subst Use Misuse 2009; 44: 1916-25.

$11 \mathrm{Rø}$ IKE, Gude T, Aasland OG. Does a self-referral counselling program reach doctors in need of help? A comparison with the general Norwegian doctor workforce. BMC Public Health 2007; 7: 36.

12 Rohlfs I, Arrizabalaga P, Artazcoz L, Borrell C, Fuentes M, Valls C. Health, Lifestyles and Working Conditions of Male and Female Doctors in Catalonia. Fundacio Galatea, 2007 (http://www.fgalatea.org).

13 Evans CE, Mellor-Clark J, Margison F. Clinical outcomes in routine evaluation: the CORE Outcome Measure (CORE-OM). J Ment Health 2000; 9: 247-55.

14 Maslach C, Jackson SE. The measurement of experienced burn-out. J Occ Behav 1981; 2: 99-113.

15 Harrison J. Doctors' health and fitness to practise: the need for abespoke model of assessment. Occup Med 2008; 58: 323-7.

16 Hochwalder J, Begsten Brucefors A. Psychological empowerment at the workplace as a predicator of ill health. Pers Individ Dif 2005; 39: 123748.

17 Halpert E. Some aspects of the psychoanalytical psychology of physicians. Int J Psychoanal 2009; 90: 1039-56.

18 Hassan TM, Ahmed SO, White AC, Galbraith N. A postal survey of doctors' attitudes to becoming mentally ill. Clin Med 2009; 9: 327-32 (http://findarticles.com/p/articles/mi_7505/is_200908/ai_n39230703/). 